\title{
The Role of Coenzyme Q10 against Cyclosporine-A Induced Cardiotoxicity in Adult Male Albino Rats (Histological and Toxicological Study)
}

\author{
Gehan A. Ahmed, Siam M.G., Sahar F. Shaban², and Ibrahim A. Awwad3 \\ ${ }^{1}$ Forensic Medicine and Clinical Toxicology Department, Faculty of Medicine, Zagazig University, Zagazig, Egypt
${ }^{2}$ Histology and Cell Biology Department, Faculty of Medicine, Zagazig University, Zagazig, Egypt
${ }^{3}$ Pharmacology Department, Faculty of Medicine, Zagazig University, Zagazig, Egypt; College of pharmacy, Qassim
University, KSA
}

\begin{abstract}
Cyclosporine-A (CsA) has a potent immunosuppressive activity and is commonly used as anti-rejection drug after organ transplantation and for treatment of some autoimmune diseases. Several toxic effects on the cardiovascular system have been reported with CsA therapy. The toxicity induced by CsA is attributed to the formation of free oxygen radicals. Coenzyme $\mathrm{Q} 10\left(\mathrm{CoQ}_{10}\right)$ is a fat soluble, vitamin-like benzoquinone compound that functions primarily as an antioxidant, and a cofactor in the oxidative phosphorylation processes. The level of $\mathrm{CoQ}_{10}$ is reduced under the conditions of illness for example after renal transplantation. The present study was done aiming to detect the possible morphological and structural changes that may occur in the cardiac muscle of the adult male albino rats duo to CsA therapy and to test the protective effect of $\mathrm{CoQ}_{10}$ against $\mathrm{CsA}$-induced cardiotoxicity. Thirty adult male albino rats were used in this study. They were classified into 3 groups and orally given the following materials for 28 days: Group (1) (Control group): that was further subdivided into 2 equal subgroups, each of 5 animals: subgroup 1a: received olive oil $1 \mathrm{ml} / \mathrm{kg}$. Subgroup 1b: (CoQ 10 group): received $5 \mathrm{mg} / \mathrm{kg} / \mathrm{day}$ of $\mathrm{CoQ}_{10}$. Group (2) (CsA-treated group): The rats received $25 \mathrm{mg} / \mathrm{kg} /$ day of CsA. Group (3) $\left(\mathrm{CoQ}_{10} \&\right.$ CsA treated group): The rats were treated with the previously mentioned doses of both $\mathrm{CoQ}_{10}$ and CsA. Systolic blood pressure (SBP), diastolic blood pressure (DBP), and heart rate (HR) were recorded three times per week. At the time of sacrifice, the hearts were dissected out and processed for light and electron microscopic examination. Oral administration of $\mathrm{CsA}$, produced a significant $(\mathrm{p}<0.05)$ elevation in SBP, DBP, and HR in comparison to the control group. Treatment with $\mathrm{CoQ}_{10}$ significantly $(p<0.05)$ reduced the increments in arterial blood pressure and heart rate. However, the recorded values were still significantly $(p<0.05)$ higher than that of the control group. The light microscopic study of sections of cardiac muscle of CsA-treated adult male albino rats showed increase in the number of infiltrated cells and disorganization of myocardial fibers with increase in the amount of connective tissue and interstitial fibrosis with perinuclear cytoplasmic vacuolation and decrease in the amount of myofilaments. Electron microscopy showed distension of the $Z$ lines with disintegration of some of them, loss of some microfilaments and disorganized intercalated discs. CoQ $\mathrm{Q}_{10}$ partially prevented most of the pathological changes revealed by the light and electron microscope. From the above mentioned results we could conclude that treatment with $\mathrm{CoQ}_{10}$ partially prevented the CsA-induced cardiotoxicity in adult male albino rats. We could recommend using $\mathrm{CoQ}_{10}$ during $\mathrm{Cs} A$ therapy to prevent its cardiotoxicity.
\end{abstract}

Keywords cyclosporine-A, coenzyme Q10, cardiotoxicity, antioxidants

\section{Introduction}

C yclosporine A (CsA), a natural polypeptide with potent immunosuppressive activity, was derived from extracts of Topocladium inflatum gams, a member of the Fungi imperfecti family (Kahan, 1999). CsA is commonly used as anti-rejection drug for organ transplantation and for treatment of some autoimmune diseases (Ponticelli, 2005).

Several toxic effects have been reported in both transplant and nontransplant settings (autoimmune disorders), including nephrotoxicity, hepatotoxicity, 
neurotoxicity, hypertension, dyslipidemia, gingival hyperplasia, hypertrichosis, malignancies and increased risk of cardiovascular toxicity (Olyaei et al., 2001).

Although mechanisms by which CsA-induced toxicity are still not clear, the former clinical and experimental findings have suggested that formation of free oxygen radicals, lipid peroxidation, and induction of cytochrome P-450 system and oxidative stress is a possible pathway of CsA-induced toxicity (Sanchez et al, 2001 and Bianchi et al, 2003).

Oxidative stress is defined as a tissue injury due to imbalance between the production of oxidants and endogenous antioxidant defense systems of an organism. Oxidants include reactive oxygen species (ROS), reactive nitrogen species, sulpher-centered radicals and others. The important cellular sources of oxidative stresses are respiratory chain of mitochondria and the oxidative burst mediated by NADPH oxidase and Nitric Oxide (NO) (Abuja and Albertini, 2001). On the other hand super oxide dismutase, catalase, glutathione peroxidase and cytochrome-c oxidase are endogenous antioxidants which play a role in the prevention of oxidative injury (Akyol et al, 2002).

Coenzyme Q10 $\left(\mathrm{CoQ}_{10}\right)$ is a fat soluble, vitamin-like benzoquinone compound that functions primarily as an endogenous antioxidant, membrane stabilizer, and a cofactor in the oxidative phosphorylation processes. The rational for the use of $\mathrm{CoQ}_{10}$ as a medical agent for treating cardiovascular diseases is based on its fundamental role in mitochondrial function and cellular bioenergetics (Ochiai et al, 2007).

The level of $\mathrm{CoQ}_{10}$ is reduced with increasing age and under the conditions of illness, for example after renal transplantation (Beyer, 1990).

Since, the myocardial protection during the CsA treatment should be remained the goal to enhance the beneficial effects of the drug as well as to remove the risk of cardiac problems, the present study was done aiming to detect the possible morphological and structural changes that may occur in the cardiac muscle of the adult male albino rats due to CsA therapy and to test the effect of $\mathrm{CoQ}_{10}$ against CsA-induced cardiotoxicity.

\section{Materials and methods}

Thirty adult male albino rats, of local strain, weighing 180-200 mg were used in this study. Female rats were excluded from the study to avoid the hormonal fluctuation during the menstrual cycle, which may influence the drug metabolism. They were housed in stainless steel cages and maintained at room temperature with a 12 hour light-dark cycle and received a standard food and water ad libitum. They were randomly classified into 3 groups (10 rats each):

- Group (I) (Control group): that was further subdivided into 2 equal subgroups, each of 5 animals: subgroup 1a: received olive oil (vehicle of $\mathrm{CoQ}_{10}$ and $\mathrm{CsA}$ ), $1 \mathrm{ml} / \mathrm{kg}$ orally for 28 days. Subgroup 1b: (CoQ 10 group): received $5 \mathrm{mg} / \mathrm{kg} /$ day of $\mathrm{CoQ}_{10}$ orally for 28 days (Crane, 2008).

- Group (II) (CsA- treated group): The rats received $25 \mathrm{mg} / \mathrm{kg} /$ day CsA orally for 28 days (Ebru et al, 2008).

- Group (III) $\left(\mathrm{CoQ}_{10}\right.$ \& CsA treated group): The rats received the above mentioned doses of both $\mathrm{CoQ}_{10}$ and CsA orally for 28 days.

$\mathrm{CoQ}_{10}$ (Co-enzyme $\mathrm{Q}_{10}$ of Mepaco Pharmaceutical Company) was available in the form of capsules $(30 \mathrm{mg} / \mathrm{capsule}) . \mathrm{CoQ}_{10}$ solution was prepared by dissolving the powder of the capsule in olive oil to a final concentration of $10 \mathrm{mg}$ of $\mathrm{CoQ}_{10}$ per ml. CsA (Sandimmun, of Novartis Pharmaceutical Company) was obtained in the form of capsules ( $25 \mathrm{mg}$ /capsule). The content of each capsule was dissolved in $5 \mathrm{ml}$ olive oil. $\mathrm{CoQ}_{10}$ and $\mathrm{CsA}$ were administrated orally by gastric tube.

Systolic blood pressure (SBP), diastolic blood pressure (DBP), and heart rate (HR) were recorded three times per week by the noninvasive method of tail-cuff plethymography (Indirect Blood Pressure Controller) in conscious rats. All procedures were performed around 1:00 p.m. to avoid any potential circadian influences (Rezzani et al, 2006).

At the time of sacrifice, all the animals were anesthetized by ether inhalation. Their hearts were carefully dissected out and their left ventricles were taken and a longitudinal cut was performed and half of each ventricle was processed for light microscopic study and the other for electron microscope. Specimens for light microscopic examination were fixed in $10 \%$ neutral formal saline for 24 hours and were processed to prepare $5 \mu \mathrm{m}$ thick paraffin sections for Haematoxylin and Eosin (H\&E) and Mallory's trichrome (Bancroft and Gamble, 2002). For electron microscope study; small myocardial biopsies $\left(1 \mathrm{~mm}^{3}\right)$ were taken, fixed in $2 \%$ glutaraldehyde and post fixed in $1 \%$ osmium tetroxide, dehydrated and embedded in epoxy resin. One $\mu \mathrm{m}$ sections (semithin sections) were preliminary obtained and stained with toluidine blue (Kiernan, 2000). Ultrathin sections were cut and double stained with uranyle acetate and lead citrate and examined by JEOL JEM 1010 transmission electron microscope in Electron Microscope Research Laboratory (EMRL), Histology Department, Faculty of Medicine, Zagazig University (Glauret and Lewis, 1998).

\section{Statistical analysis}

The obtained data of SBP, DBP and HR of all groups were subjected to one-way ANOVA. The mean difference was considered significant at $p<0.05$.

\section{Results}

\section{Blood pressure and heart rate results}

Oral administration of $\mathrm{CsA}$, produced a significant $(p<0.05)$ elevation in SBP, DBP, and HR in comparison with the control group. Treatment with $\mathrm{CoQ}_{10}$ significantly $(\mathrm{p}<0.05)$ reduced the increments in SBP, DBP and HR in comparison with CsA-treated rat group. However, the recorded values were still 
significantly $(\mathrm{p}<0.05)$ higher than that of the control group, Table (1) and Chart (1).

\section{Histological Results I- Light microscopic results}

\section{A- Haematoxylin and Eosin stain}

Examination of H\&E stained sections of the control group (subgroup $1 \mathrm{a}$ and $1 \mathrm{~b}$ ) revealed the classical architecture of the cardiac muscle fibers. They appeared arranged in a linear array that branch and anastomose giving the appearance of a sheet. They contained acidophilic sarcoplasm with oval, single, centrally located nuclei (Fig.1).

H\&E stained myocardial sections of CsAtreated rats (group II) revealed apparent disorganization of the muscle fibers. They exhibited areas of myofibrillar degeneration accompanied with small deeply stained nuclei. Moreover, interstitial cellular infiltration was recognized (Fig.2). These changes were also associated with presence of extravasated red blood cells among muscle cells as well as wide interstitial spaces were revealed (Fig.3)

Co-administration of CoQ10 and CsA showed that most of the muscle fibers exhibited nearly normal appearance. However, most of their nuclei were centrally located but darkly stained. In addition, few sarcoplasmic vacuoles were still persistent (Fig.4).

\section{B- Mallory's trichrome stain}

The cardiac muscle of the control group (subgroup1a \&1b) revealed delicate collagen fibers in the endomysium (Fig.5). On the other hand, sections of CsA-treated rat (group II) exhibited increase deposition of collagen fibers in the interstitial spaces (Fig.6). Whereas, upon combination of CoQ10 and CsA, fine and delicate collagen fibers deposition among cardiac myocytes was noticed (Fig.7).

\section{C- Toluidine blue stain}

Examination of semithin sections of cardiac muscle fibers of control rats (subgroupla \&1b) revealed polygonal appearance of the muscle cells with centrally located nuclei and darkly stained mitochondria were appeared. Normal blood capillaries were found in the endomysium (Fig.8). However, semithin sections in myocardium of CsA-treated rat (group II) depicted sarcoplasmic vacuoles and peripherally located nuclei. In addition, interstitial blood vessels were congested (Fig.9). While, simultaneous administration of CoQ10 and CsA revealed that most of cardiac myocytes retained nearly normal appearance with centrally located nuclei and dark stained mitochondria (Fig.10).

\section{II- Electron microscopic results}

Electron microscopic examination of cardiac muscle fibers of control rats (subgroup1a \&1b) revealed its normal ultrastructure. Cardiac myocytes exhibited single central oval euchromatic nuclei with prominent nucleoli. Their sarcoplasm appeared packed with longitudinally arranged myofibrils showing its cross banding pattern that oriented into sarcomeres. Numerous mitochondria were arranged in rows between the myofibrils. Cardiac myocytes attached end to end by intercalated discs. Each disc consisted of a stepwise arrangement of longitudinal and transverse components. The longitudinal (lateral) portion was parallel to the myofibrils while the transverse portion was localized at the level of Z-line (Fig.11).

Examination of the myocardium of CsAtreated rats (group II) revealed obvious ultrastructural disturbances. Most of the cardiac myocytes showed loss of normal pattern of the myofibrils with shorting of sarcomeres. As well as some myofibrillar fragmentation and sarcoplasmic vacuolation were apparent. Disorganized mitochondria were also observed and were displayed in groups. Most of nuclei appeared electron dense with condensation of clumps of heterochromatin. In addition disruption of intercalated discs were depicted (Fig.12). These changes were associated with inflammatory cellular infiltration and increase deposition of collagen fibers in the interstitial spaces (Figs. 13\&14).

Co-administration of $\mathrm{CoQ}_{10}$ and CsA reflected a considerable degree of decreased myocardial changes of CsA insults. As most of the cardiac myocytes showed regular pattern of striation with well organized sarcomeres, relatively regular arranged mitochondria and regular pattern of intercalated discs. Euchromatic nuclei were also obvious. However, some cardiac muscle cells still exhibited interruption and fragmentation of myofibrils as well as bizarre shaped mitochondria (Figs. 15, $16 \& 17$ ).

Table (1): The effect of CsA, $\mathrm{CoQ}_{10}$, and their combination on SBP, DBP and HR in adult male albino rats in comparison to the control group.

\begin{tabular}{|c|c|c|c|c|}
\hline \multirow{2}{*}{ Mean \pm SD } & \multicolumn{2}{|c|}{ Control group } & \multirow{2}{*}{$\begin{array}{c}\text { CsA-Treated } \\
\text { Rats }\end{array}$} & \multirow{2}{*}{$\begin{array}{l}\mathrm{CoQ}_{10}+\mathrm{CsA} \\
\text { Treated Rats }\end{array}$} \\
\hline & Subgroup 1a & Subgroup 1b & & \\
\hline Systolic blood pressure & $117 \pm 4.79^{(\mathrm{a})}$ & $115 \pm 4.50^{(\mathrm{a})}$ & $142 \pm 4.57^{(b)}$ & $131 \pm 5.79^{(\mathrm{c})}$ \\
\hline Diastolic blood pressure & $76 \pm 4.79^{(\mathrm{a})}$ & $70 \pm 3.99^{(a)}$ & $145 \pm 13.32^{(b)}$ & $115 \pm 4.08^{(\mathrm{c})}$ \\
\hline Heart rate & $253 \pm 17.08^{(a)}$ & $245 \pm 18.32^{(a)}$ & $345 \pm 24.49^{(b)}$ & $294 \pm 11.09^{(\mathrm{c})}$ \\
\hline
\end{tabular}

Values (Means) with different superscript letters are significantly different by one way ANOVA $(P<0.05)$. 


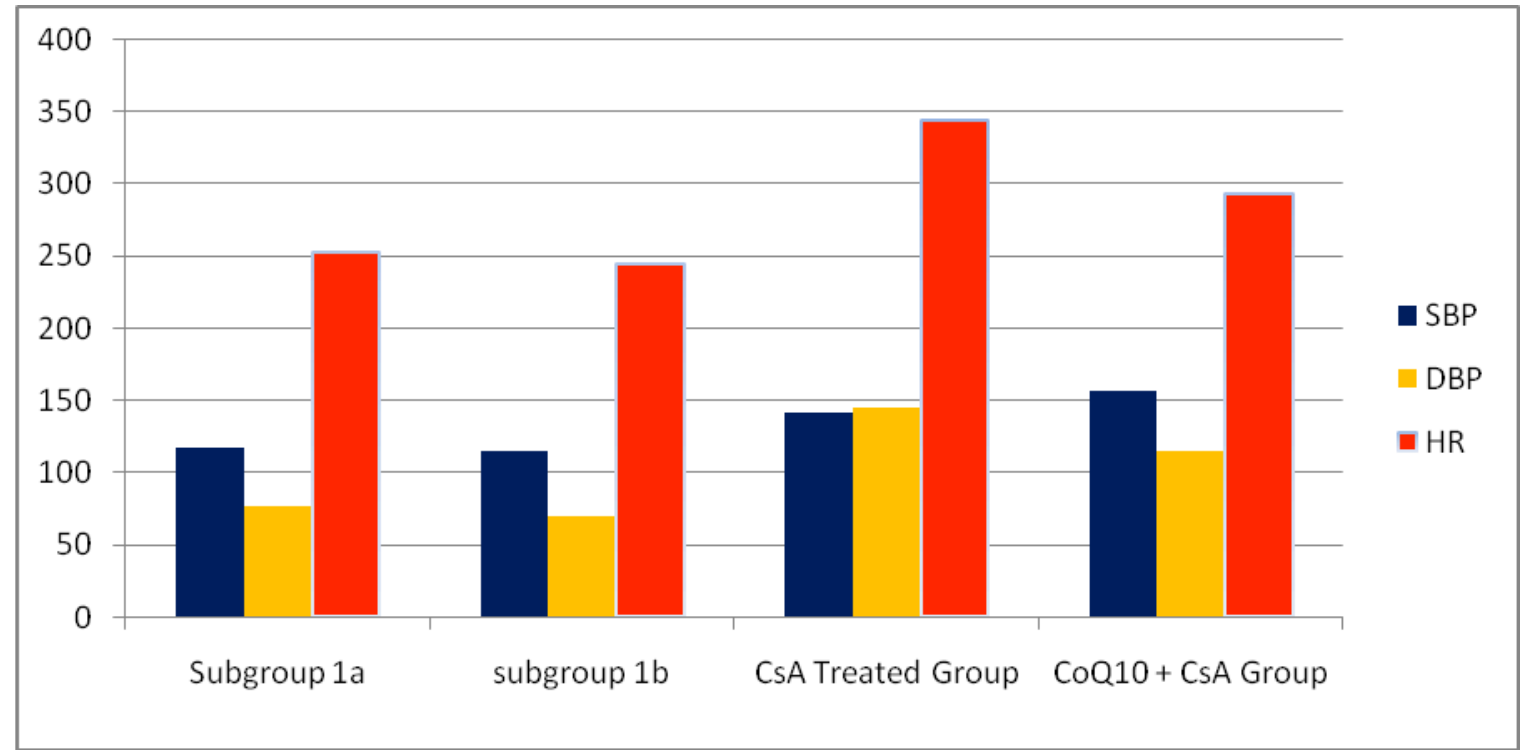

Chart (1): The effect of CsA, CoQ10, and their combination on SBP, DBP and HR in adult male albino rats in comparison to the control group.

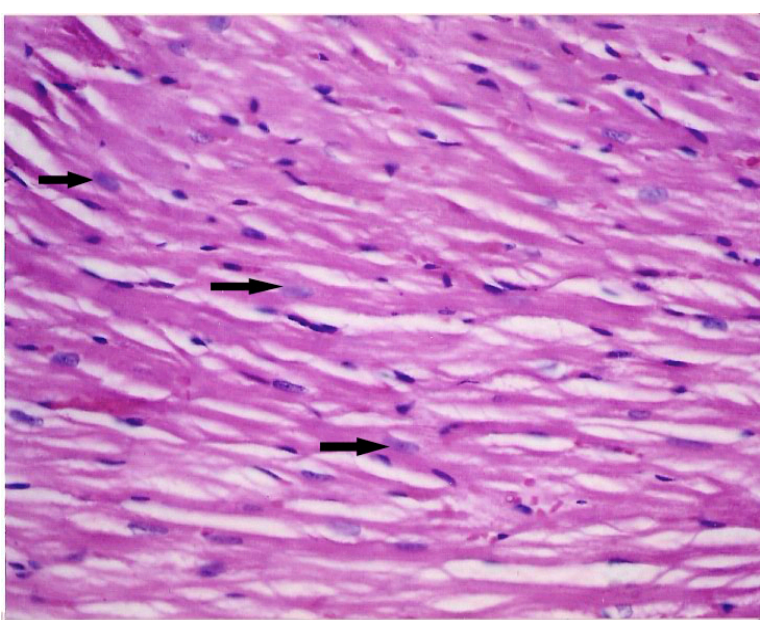

Fig. (1): A photomicrograph of a section in the cardiac muscle of a control group showing the characteristic branching and interconnection pattern of the muscle fibers that are sectioned longitudinally with acidophilic cytoplasm and centrally located nuclei (arrow). (H\&E X400).

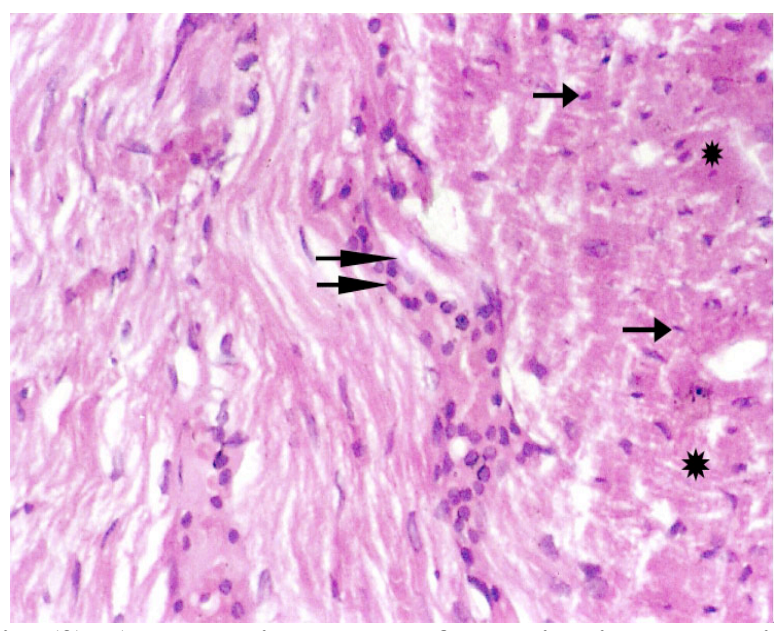

Fig. (2): A photomicrograph of a section in the cardiac muscle of CsA-treated group showing disorganization of the muscle fibers with small deeply stained nuclei (arrow) and areas of myofibrillar degeneration (*). Interstitial cellular infiltration (double arrows) among cardiac muscle cells are noticed. (H\&E X400). 


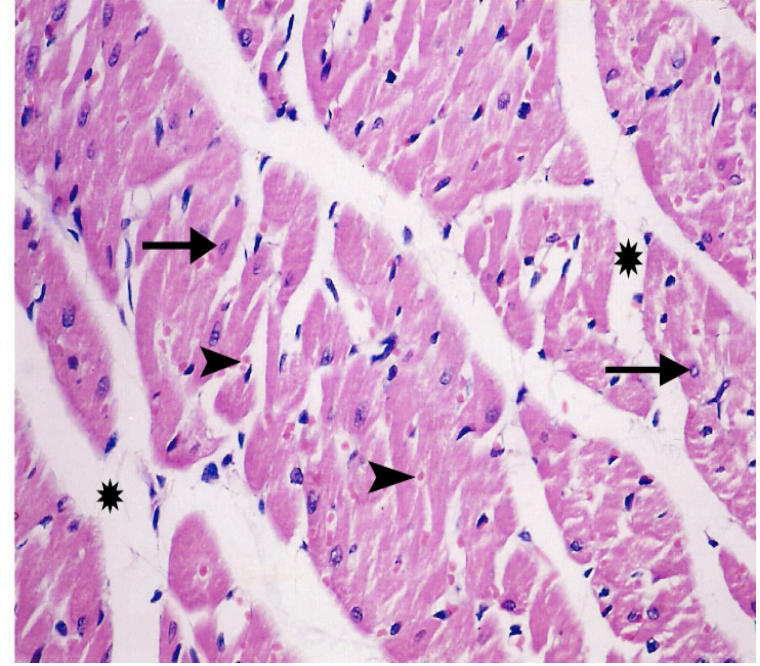

Fig. (3): A photomicrograph of a section in the cardiac muscle of CsA-treated group showing extravasated red blood cells (arrow head) in-between cardiac muscle cells. Many cardiac muscle cells have small dark stained nuclei (arrow). Wide interstitial spaces are recognized (*) (H\&E X400).

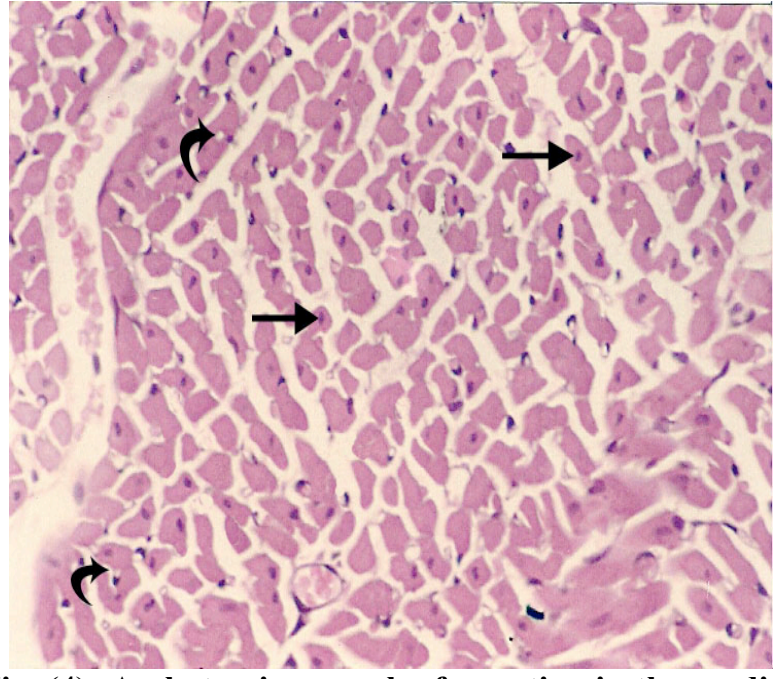

Fig. (4): A photomicrograph of a section in the cardiac muscle of a rat treated with both $\mathrm{CoQ}_{10}$ and $\mathrm{CsA}$ showing cardiac muscle fibers cut transversely with most of their nuclei are dark and centrally located (arrow). Few sarcoplasmic vacuoles are noticed (curved arrow). (H\&E X400).

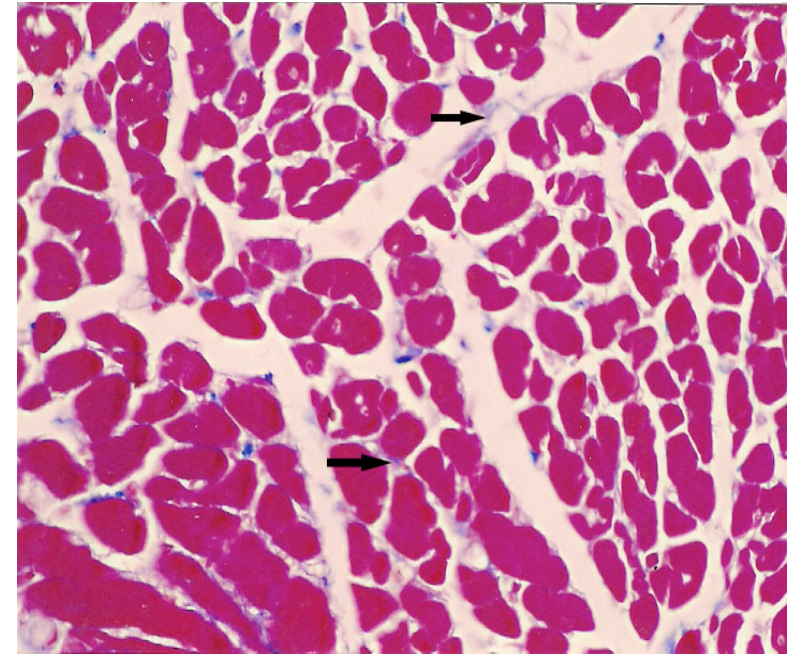

Fig. (5): A photomicrograph of a section in the cardiac muscle of a control group showing transversely cut muscle fibers separated by delicate collagen fibers (arrow) distributed in the endomysium. (Mallory's trichrome X400).

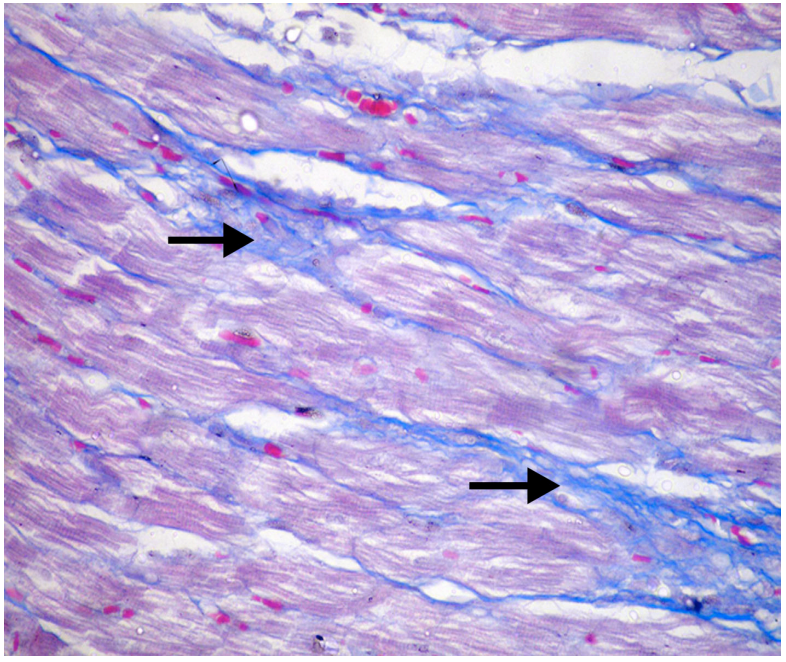

Fig. (6): A photomicrograph of a section in the cardiac muscle of CsA-treated group showing intense deposition of collagen fibers (arrow) distributed in the endomysium among myocardial fibers. (Mallory's trichrome X400). 


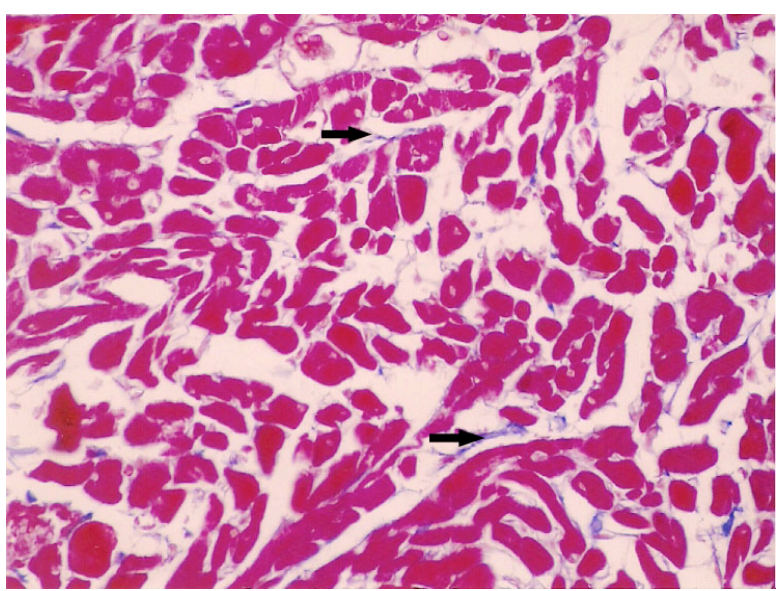

Fig. (7): A photomicrograph of a section in the cardiac muscle of a rat treated with both $\mathrm{CoQ}_{10}$ and CsA showing cardiac muscle fibers separated by delicate collagen fibers (arrow) distributed in the endomysium. (Mallory's trichrome; X400).

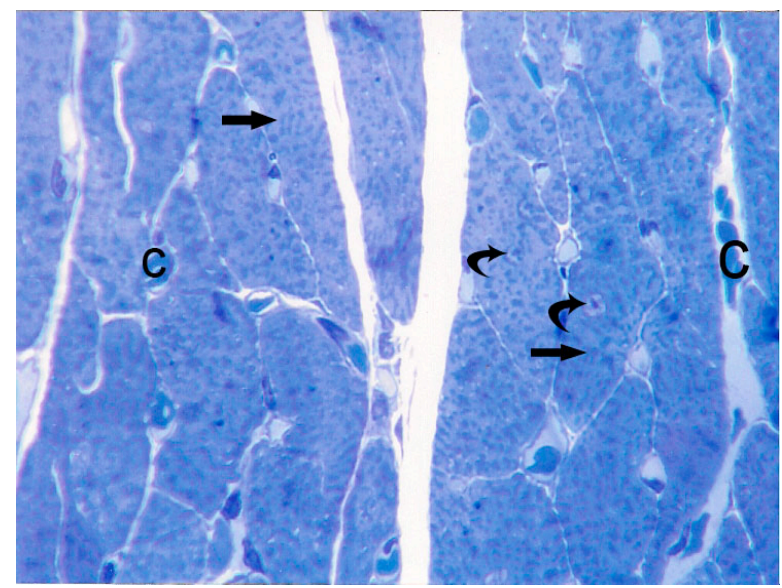

Fig. (8): A photomicrograph of a semithin section $(1 \mu \mathrm{m}$ thick) in the cardiac muscle of a control group showing polygonal appearance of cardiac muscle fibers with centrally located nuclei (curved arrow). Mitochondria appear as deeply stained dots (arrow). Blood capillaries (c) are also noticed in the endomysium. (Toluidine blue X1000).

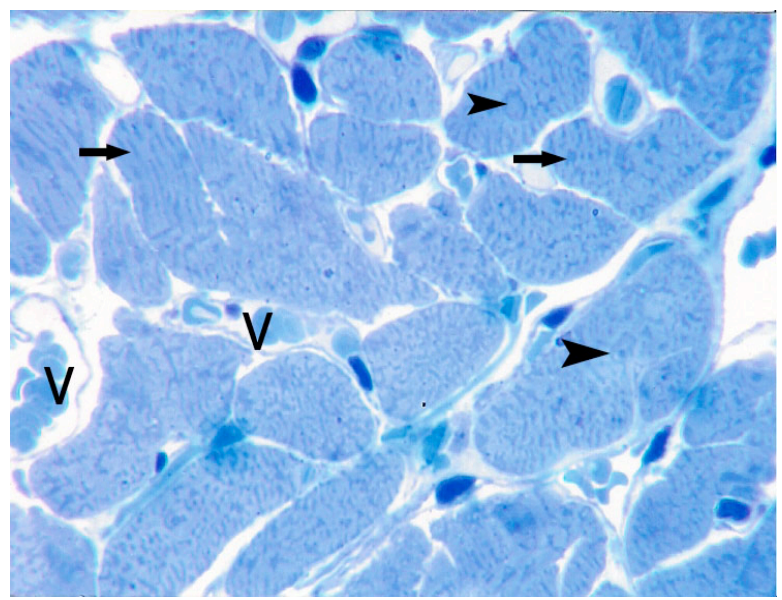

Fig. (10): A photomicrograph of a semithin section in the cardiac muscle of a rat treated with both $\mathrm{CoQ}_{10}$ and CsA showing that cardiac myocytes cut transversely with centrally located nuclei (arrow head). Their sarcoplasm exhibit deeply stained dots represent mitochondria (arrow). Blood vessels are seen in the endomysium (v). (Toluidine blue X1000). 


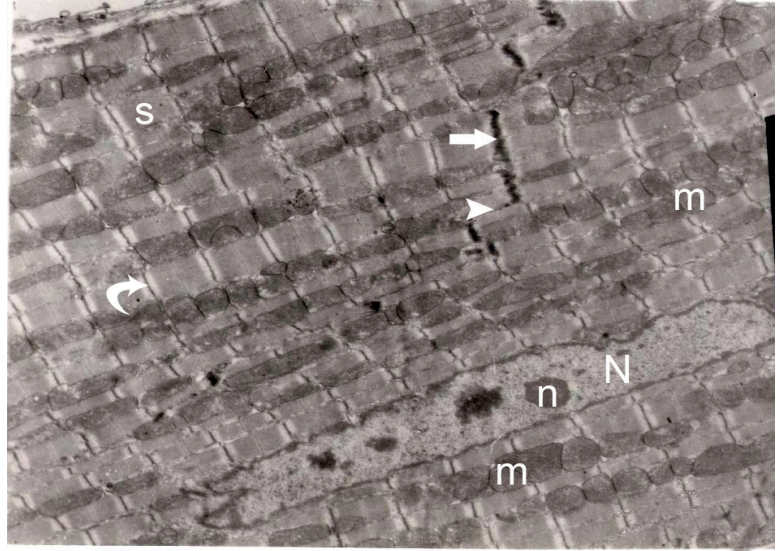

Fig. (11): An electron micrograph from cardiac muscle of a control group showing a part of cardiac muscle cell with oval euchromatic nucleus $(N)$ with prominent nucleolus (n). Its sarcoplasm is packed with longitudinally arranged myofibrils that are oriented into sarcomeres (s). Mitochondria are arranged in rows between the myofibrils (m). Intercalated disc is found between cardiac myocytes with its transverse portion (arrow) at Z-line (curved arrow) and its lateral (longitudinal) portion parallel to the myofibrils (arrow head). (X4000)

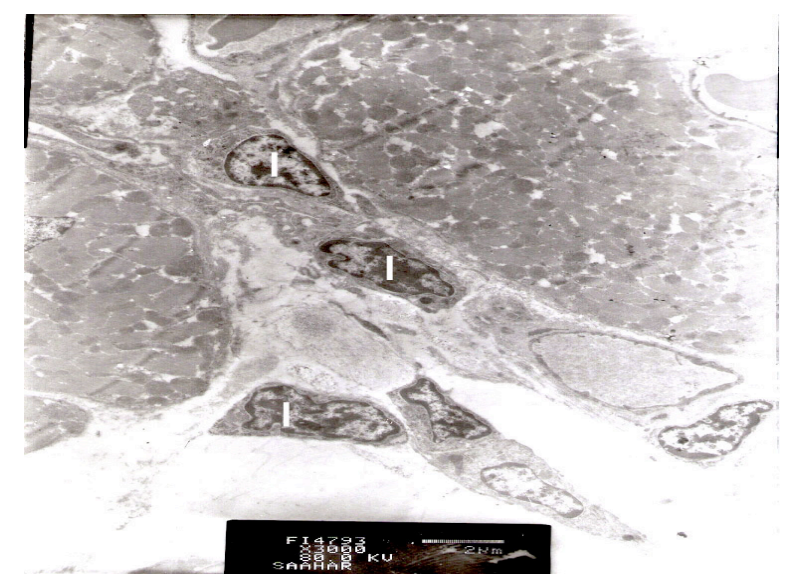

Fig. (13): An electron micrograph of cardiac muscle of CsA-treated group showing apparent disturbance of usual architecture of cardiac myocytes with inflammatory cellular infiltration (I) among them. (X3000)

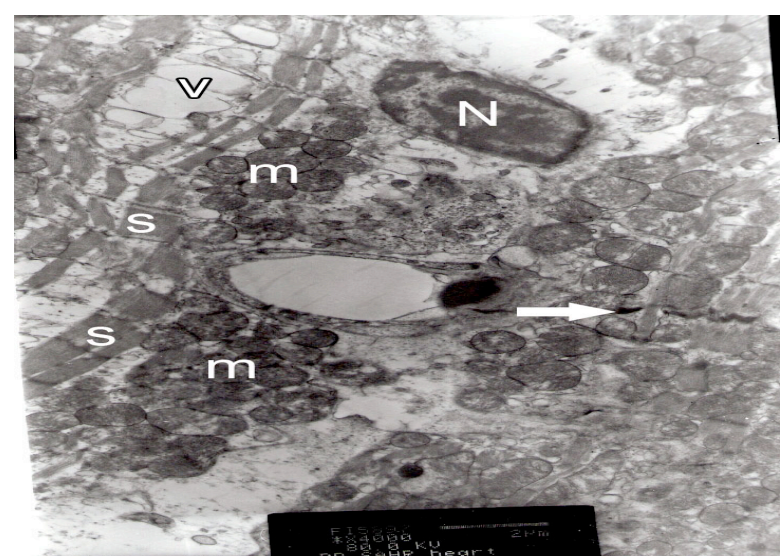

Fig. (12): An electron micrograph of cardiac muscle of CsA-treated group showing apparent loss of normal architecture of the cardiac myofibrils with shorting of sarcomeres (s) and disorganization of mitochondria (m) which present in groups. Myofibrillar fragmentation and sarcoplasmic vacuolations are also noticed (v). Intercalated disc appears disrupted (arrow) and electron dense nucleus with clumps of heterochromatin $(\mathrm{N})$ is seen. (X4000)

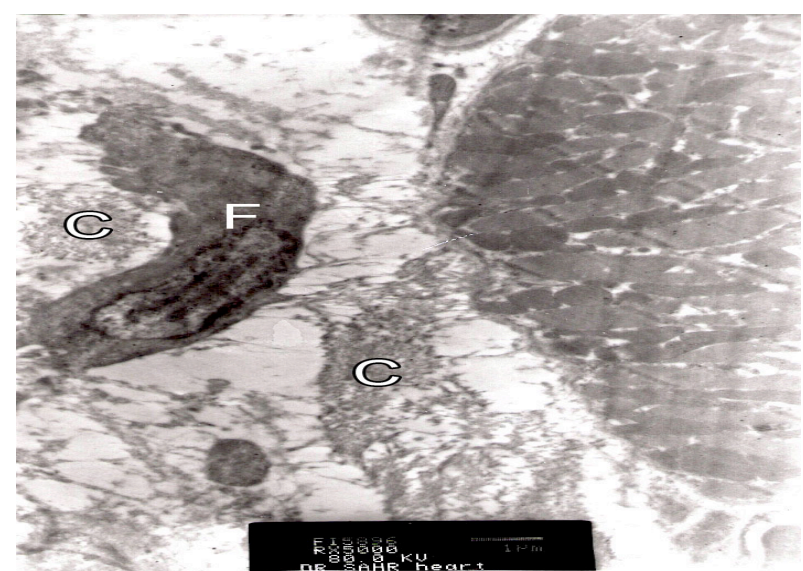

Fig. (14): An electron micrograph of cardiac muscle of CsA-treated group showing excess collagen fibers (C) close to a part of cardiac myocyte and fibroblast is also seen (F). (X5000) 


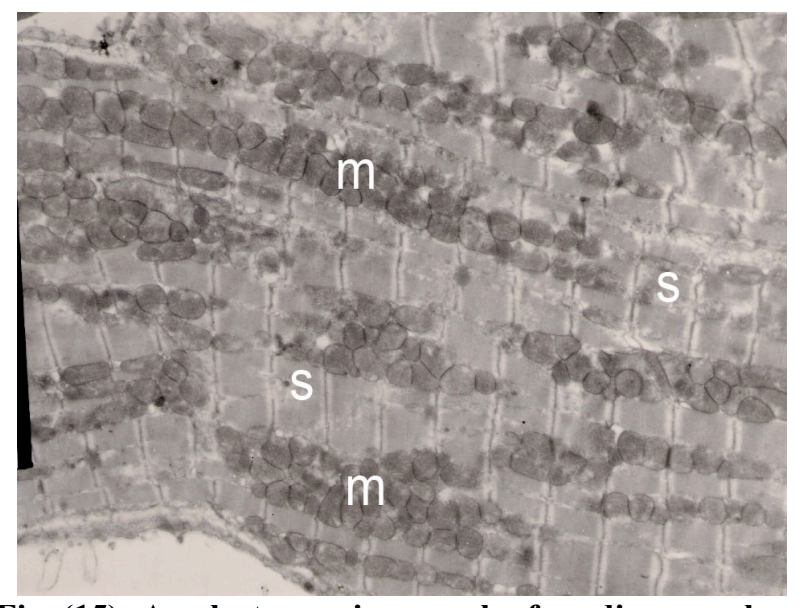

Fig. (15): An electron micrograph of cardiac muscle of a rat treated with both $\mathrm{CoQ}_{10}$ and $\mathrm{CsA}$ showing regular pattern of striation with well organized sarcomeres (s) and relative regular arranged mitochondria (m). (X4000).

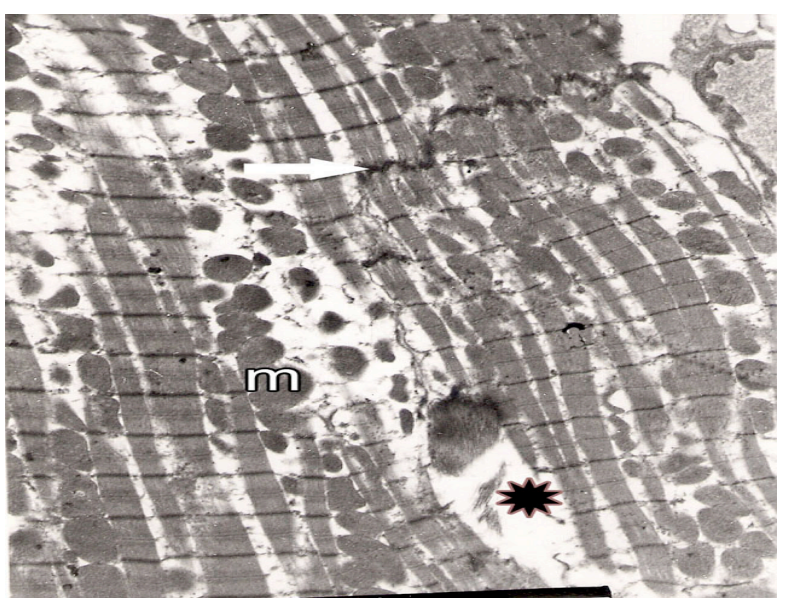

Fig. (16): An electron micrograph of cardiac muscle of a rat treated with both $\mathrm{CoQ}_{10}$ and $\mathrm{CsA}$ showing relatively regular pattern of intercalated disc (arrow) and mitochondria $(\mathrm{m})$. Some sarcomeres have interrupted and fragmented myofibrils $(*)$. (X4000)

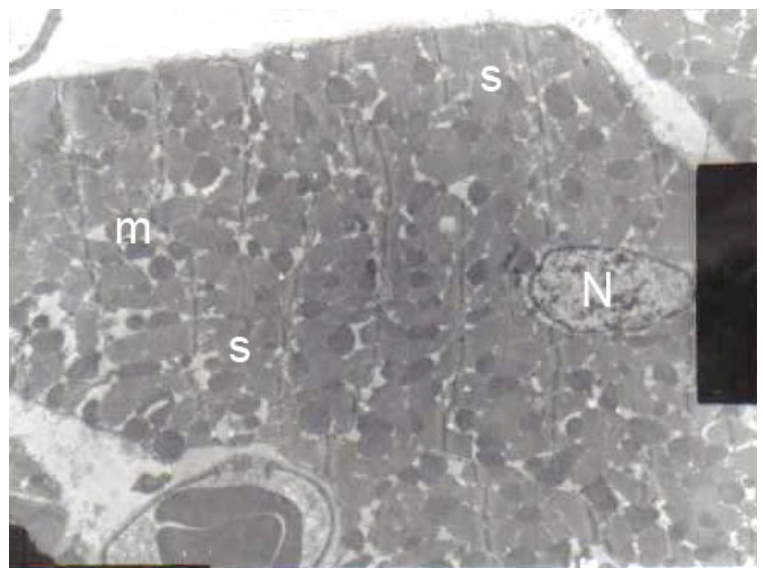

Fig. (17): An electron micrograph of cardiac muscle of a rat treated with $\mathrm{CoQ}_{10}$ and $\mathrm{CsA}$ showing relative regular pattern of striation with well organized sarcomeres (s). Bizarre shaped mitochondria (m) and euchromatic nucleus $(\mathrm{N})$ are also seen. (X6000)

\section{Discussion}

Cyclosporin-A (CsA) is the most frequently used immune-suppressant in transplant surgery and in the treatment of autoimmune diseases. It has been shown that CsA is able to generate reactive oxygen species and lipid peroxidation, which are directly involved in the CsA-induced cardiotoxicity. The cardiovascular complication remains the main cause of post transplantation mortality (Reis et al, 2007). So, the aim of this work was to study the blood pressure and heart rate changes as well as the histopathological changes in the cardiac muscles after administration of CsA in adult male albino rats for 28 days. We aimed also to study the efficacy of $\mathrm{CoQ}_{10}$ as a protector against CsAinduced cardiotoxicity.

In the present work, administration of CsA in a dose of $25 \mathrm{mg} / \mathrm{kg} /$ day for 28 days induced significant elevation in SBP, DBP, and HR in comparison with the control group. These findings are consistent with Oriji and Schanz, 2001, who reported that CsA increases the mean arterial pressure in normotensive rats.

CsA induced hypertension could be attributed to several mechanisms: Activation of rennin angiotensin system, enhancement of $\alpha-1$ adrenoceptor affinity to noradrenalin and their related transduction mechanisms including increase in calcium influx and inositol phosphate turn over, and activation of protein kinase (Oriji and Schanz, 2001).

Throughout the present study, CsA induced apparent increase in the heart rate that was in accordance with the report of Omar and El-Mas, 2004, who attributed this effect to the attenuation of vagal activity and enhancement of sympathetic activity by CsA. Furthermore, Florio et al, 2003, reported that CsA increases calcium influx $\left(\mathrm{Ca}^{++}\right.$overload $)$and enhances 
lipid peroxidation in cardiomyocytes leading to cardiotoxicity.

In the present work, administration of $\mathrm{CoQ}_{10}$ in a dose of $5 \mathrm{mg} / \mathrm{kg} /$ day along with CsA produced significant decrease in the CsA-induced elevation in arterial blood pressure and heart rate as compared to the rats received CsA alone. However, the recorded values were still significantly higher than that of the control group. Tran et al, 2001, reviewed eight different trials that documented the decrease in blood pressure following different doses of $\mathrm{CoQ}_{10}$.

Systemic reviews and meta-analysis have been conducted evaluating $\mathrm{CoQ}_{10}$ in hypertension versus placebo. Decrease in SBP was found in some patients. However, confounding variables, small trial size, and variable study designs make extrapolation of the data difficult (Pepe et al, 2007).

Rezzani et al, 2006, showed comparable results when using melatonin as a cardio protective agent against $\mathrm{CsA}$-induced hypertension.

Histological results of the current work clearly demonstrated that CsA administration induced prominent myocardial changes in the form of disorganization of the muscle fibers with areas of myofibrillar degeneration accompanied with small deeply stained, peripherally located nuclei. Intense cellular infiltration and areas of increased collagen fibers deposition were revealed as well. Ultrastructurally, apparent changes involving the myofibrils, mitochondria, intercalated discs and nuclei were encountered. These results were in agreement with Bianchi et al, 2003; Rezzani, 2004 and Selcoki et $\mathrm{al}, 2007$. The results of the previous researches indicate that myocardial changes involved both the atria and ventricles in CsA treated rats.

In the present study, myofibrillar affections included shortened sarcomeres, myofibrillar fragmentations and sarcoplasmic vacuolations. This change could be attributed to $\mathrm{Ca}^{++}$overload that enhances the contractile forces within the muscle (Florio et al, 2003). The previous researchers demonstrated an evident increase in intracellular $\mathrm{Ca}^{++}$ in rat cardiomyocytes in a dose-dependent manner following CsA-treatment. They suggested that CsA toxicity might be attributed to $\mathrm{Ca}^{++}$overload that in turn, induced lipid peroxidation and consequent cell injury.

Mitochondria were among the affected organelles. They were frequently seen disorganized with loss of normal orientation and present in groups. Such mitochondrial alterations could be attributed to elevated intracellular calcium accumulation and consequent lipid peroxidation of mitochondrial membranes. The effect occurs when ROS attack the double bond of unsaturated fatty acids leading to formation of peroxides that lead to propagation of extensive membrane damage. This might also explain the observed of the intercalated discs. This interpretation was supported by Molkentin (2001) who stated that CsA is one of the direct effectors of mitochondrial integrity. He added that CsA act as a potent suppressor of membrane destabilization and subsequent initiation of apoptosis.

Throughout the present study, prominent nuclear changes were in the form of dark, small, and condensation of clumps of heterochromatin (apoptotic nuclei). Cardiac myocytes of CsA-treated rats displayed peripherally located nuclei during light microscopic examination. This was explained by the fact that desmin cytoskeletal intermediate filaments form a sarcoplasmic network radiating from the sarcolemma to the nuclear surface close to the nuclear pores which is related to lamin B nucleoskeletal intermediate filaments. Disruption of this cytoskeleton by stimulation of protease enzymes secondary to disrupted calcium homeostasis due to CsA administration would be responsible for nuclear displacement of cardiac myocytes (Stacchiotti et al., 2009).

One of the prominent changes observed in most of cardiac sections of CsA treated group were congestion, dilatation of blood vessels and extravasations of blood cells in the wide interstitium. These observations were consistent with Yüce et al, 2008. These changes were mostly explained by increase nitric oxide (NO) production which is endothelium relaxation factor with subsequent vasodilatation. $\mathrm{NO}$ is a free radical molecule that plays a key role in CsA-induced myocardial injury. In support for this explanation, high NO production has been demonstrated in the myocardium during CsA therapy (Ebru et al., 2008).

Interstitial fibrosis encountered in the CsAtreated group could be explained on the basis of the fact that ROS production activates matrix metalloproteinase enzymes and induces a decrease in their endogenous inhibitors with imbalance between matrix deposition and degradation (Siwik et al, 2001). They reported dysregulation of the myocardial extracellular matrix in different cardiomyopaties, upregulation of metalloproteinases with inhibition of endogenous inhibitors systems.

Bianchi et al, 2003, observed that after CsA treatment, there is increase in the metalloproteinase activity and the myocardial extracellular matrix. They also reported the occurrence of disorganization and disappearance of the normal network among fibers. 
Regarding the mechanisms by which CsA induces cardiotoxicity, it has not been documented in detail yet and still a matter of debate. In fact, it seems that CsA can cause excess free radical production during its metabolism, and/or impair antioxidant system. These free radicals could activate several protein kinases in both myocytes and fibroblasts. This mechanism has already been considered as a pathway involved in induction of cardiac fibrosis (Siwik and Colucci, 2004). The second hypothesis, supposed an indirect action of CsA on ROS, through the decrease of cytochrome-c oxidase activity, which is an endogenous antioxidant marker (Yang and Dettbarn, 1998).

Kim et al, 2002, documented that ROS produces cell and tissue damage after CsA administration. Nangaku et al, 2005, reported that CsAinduced renal injury is associated with activation of the intra-renal complement system. The complement system is an important component of the immune system, but irregular or excess activation of the complement system can cause tissue damage.

The current work revealed that upon combination of $\mathrm{CoQ}_{10}$ and $\mathrm{CsA}$, better amelioration of CsA-induced histological alterations of the cardiac muscle was obvious. Cardiac myocytes expressed a morphological pattern similar to that of the controls except for little amount of disorganization of myocardial fibers, mitochondria and few sarcoplasmic vacuoles between myofibrils.

Since the role of free radicals in CsA cardiotoxicity is widely accepted. Therapeutic strategies are aimed for limiting free radical mediated injury. So, $\mathrm{CoQ}_{10}$ was tried in the current study. The reduced form of $\mathrm{CoQ}_{10}$ is the only endogenously synthesized lipophilic antioxidant. $\mathrm{CoQ}_{10}$ act as antioxidant directly by protecting biological membranes against oxidants, and can also inhibit the peroxidation of lipoprotein lipids present in the blood (Nishimura et al, 2009). The hydrophobic character of CsA may suggest that the most potent protector from its harmful effect on lipid membranes can be the main hydrophobic antioxidant, vitamin $\mathrm{E}$ and $\mathrm{CoQ}_{10}$ (Sawicka et al, 2006).

Sawicka et al, 2006, reported that $\mathrm{CoQ}_{10}$ appears to be more protective (given before CsA) than a reparative agent in decreasing the lipid peroxidation enhanced by CsA. Ankola et al, 2010, described the first trial to design nano-scale systems for simultaneous encapsulation of $\mathrm{CsA}$ and $\mathrm{CoQ}_{10}$. They stated that the combination therapy of both $\mathrm{CsA}$ and $\mathrm{CoQ}_{10}$ in one capsule will soon become a reality.

In parallel with our results, Ebru et al, 2008, observed that pretreatment with Nigella Sativa oil reduced the subsequent CsA-induced injury in rat's heart, demonstrated by normalized cardiac histopathology, decrease lipid peroxidation and improvement in the antioxidant enzyme status.

From the above mentioned results it could be concluded that: $\mathrm{CoQ}_{10}$ partially alleviates the CsAinduced cardiotoxicity in the form of decreasing the elevated systolic, diastolic blood pressure, and heart rate. Morever, $\mathrm{CoQ}_{10}$ reduced the occurrence of major histological changes in the cardiac muscles as evidenced by light and electron microscope. On the light of the results of this study, the use of $\mathrm{CoQ}_{10}$ as a cardioprotective against CsA-induced cardiotoxicity could be recommended.

\section{References}

Abuja PM and Albertini R (2001): Methods for monitoring oxidative stress, lipid peroxidation and oxidation resistance of lipoproteins. Clin. Chim. Acta. 306: 1-17.

Akyol O, Herken H, Ebru UZ et al., (2002): The indices of endogenous oxidative and antioxidant processes in plasma from schizophrenic patients. The possible role of oxidant/antioxidant imbalance. Prog. Neuropsychopharmacol. Biol. Psychiatr. 26: 955-1005.

Ankola DD, Durbin EW, Buxton GA et al., (2010): Preparation, characterization and in silico modeling of biodegradable nanoparticles containing cyclosporine $\mathrm{A}$ and Coenzyme Q10. Nanotechnology Vol.21, No.6 (Abst).

Bancroft J and Gamble A (2002): Theory and Practice of Histological Techniques. $5^{\text {th }}$ ed., Churchill Living Stone. New York, London.

Beyer RE (1990): The participation of co-enzyme Q in free radical production and antioxidation, Free Rad Biol Med; 8, 545-565.

Bianchi R, Rodella L and Rezzani R (2003): Cyclosporine-A up-regulates expression of matrix metalloproteinase 2 and vascular endothelial growth factor in heart rat.

Crane FL (2008): The evolution of coenzyme Q. Bio Factors, 32 (1-4): 5-11.

Ebru UZ, Burak UZ, Yusuf S et al., (2008): Cardioprotective effects of Nigella Sativa oil on cyclosporine-A induced cardiotoxicity in rats. Nordic Pharmacological Society. Basic \& Clinical Pharmacology \& Toxicology 103, 574-580.

Florio S, Ciarcia R, and Crispino L (2003): Hydrocortisone has a protective effect on cyclosporine-A induced cardiac toxicity. J Cell Physiol. Apr; 195 (1): 21-6. 
Glauret AM and Lewis PR (1998): Biophysical specimen preparation for transmission electron microscopy.Vol.17, Parhand press, London.

Kahan BD (1999): Cyclosporine: a revolution in transplantation. Transplant. Proc. 31, 14S$15 \mathrm{~S}$.

Kiernan JA (2000): Histological and Histochemical methods: theory and practice. $3^{\text {rd }}$ ed., pp $267-$ 281, 390-418.

Kim YH, Lee SH, and Mun KC (2002): Effect of melatonin on antioxidant status in the plasma of cyclosporine-treated rats. Transplant. Proc., 34: $2652-2653$

Molkentin JD (2001): Calcineurin, mitochondrial membrane potential, and cardiomyocyte apoptosis. Circulation Research, 88: 12201222.

Nangaku M, Shankland SJ and Couser WG (2005): Cellular response to injury in membranous nephropathy. J Am Soc Nephrol, May; 16 (5): 1195-204.

Nishimura A,Yanagawa H, Fujikawa N et al., (2009): Pharmacokinetic profiles of coenzyme Q10: absorption of three different formulations in rats. J of Health Science, 55 (4): 540-548.

Ochiai A, Itagaki S, and Kurokawa T (2007): Improvement in intestinal coenzyme Q10 absorption by food intake. Yakugaku Zasshi, 127, 1251-4.

Olyaei AJ, de Mattos AM, and Bennett WM (2001): Nephrotoxicity of immunosuppressive drugs: new insight and preventive strategies. Curr. Opin. Crit. Care 7, 384-389.

Omar AG and El-Mas MM (2004): Time-Domain evaluation of cyclosporine interaction with hemodynamic variability in rats. Cardiovascular Drug Therapy, Nov; 18 (6): 461-8.

Oriji GJ and Schanz N (2001): Nitric oxide in CsAinduced hypertension: role of betaadrenoceptor antagonist and thromboxane $\mathrm{A}_{2}$. Prostaglandins Leukot Essential fatty Acids, Nov-Dec; 65(5-6): 259-63.

Pepe S, Marasco SF, and Haas SJ (2007): Coenzyme Q10 in cardiovascular disease. Mitochondrion 7 (Suppl): S154-S167.

Ponticelli C (2005): Cyclosporine from renal transplantation to autoimmune disease. Ann. NY Acad. Sci.; 1051: 551-558.

Reis F, Lemos TE, Almeida L et al., (2007): Dual effect of nitrate therapy for cyclosporine A- induced hypertension on vascular and platelet morphofunctional markers: An animal model. Transplant Proceedings, 39(8):2501-6.

Rezzani R (2004): Cyclosporine A and adverse effects on organs: histochemical studies. Prog. Histochem. Cytochem.; 39: 85-128.

Rezzani R, Roddella LF, Bonomini F et al., (2006): Beneficial effects of melatonin in protecting against cyclosporine A-induced cardiotoxicity are receptor medicated. J. pineal Res. 41: 288295.

Sanchez H, Zoll J, Bigard X et al., (2001): Effect of cyclosporine $\mathrm{A}$ and its vehicle on cardiac and skeletal muscle mitochondria relationship to efficacy of the respiratory chain. $\mathrm{Br} \mathrm{J}$ pharmacol. 133(6): 781-788.

Sawicka E, Dlugosz A and Przybylska D (2006): The effect of cyclosporine $A$ on free radical processes and interactions with antioxidants. Adv Clin Exp Med 15;3, 435-444.

Selcoki Y, Ebru UZ, Bayrak R et al., (2007): The protective effect of erdosteine against cyclosporine A-induced cardiotoxicity in rats. Toxicology, 239: 53-59.

Siwik DA and Colucci WS (2004): Regulation of matrix metalloproteinases by cytokines and reactive oxygen/ nitrogen species in the myocardium. Heart Fail. Rev.9: 43-51.

Siwik DA, Pagano PJ, and Colucci WS (2001): Oxidative stress regulates collagen synthesis and matrix metalloproteinase activity in cardiac fibroblasts. Am. J. physiol. 280: C35C60.

Stacchiotti A, Bonomini F, Lavazza A et al., (2009): Adverse effects of cyclosporine A on HSP25, alphaB-crystallin and myofibrillar cytoskeleton in rat heart. Toxicology, 262 (3):192-198.

Tran MT, Michell TM, and Kennedy DT (2001): Role of Coenzyme Q10 in chronic heart failure, angina, and hypertension. Pharmacotherapy 21(7):797-806.

Yang ZP and Dettbarn WD (1998): Lipid peroxidation and changes in cytochrome c oxidase and xanthine oxidase activity in organophosphorus anticholinesterase induced myopathy. J. Physiol. Paris 92, 157-161.

Yüce A, Atessahin A and Ceribasi AO (2008): Amelioration of cyclosporine A-induced renal, hepatic and cardiac damages by ellagic acid in rats. Basic \& Clinical Pharmacology \& Toxicology, 103: 186-191. 


\section{الملخص العربي

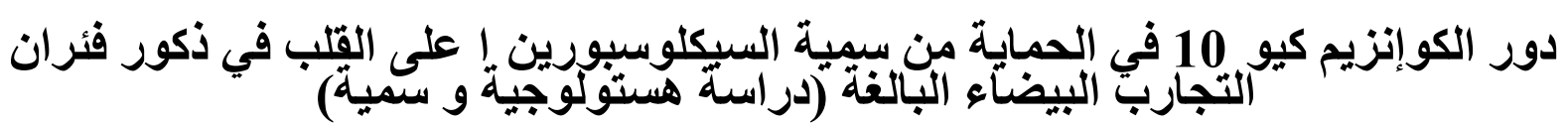

\section{جيهان أحمد و صيام م.ج. و سحر فرج شعبان مو إبراهيم علي عواد3}

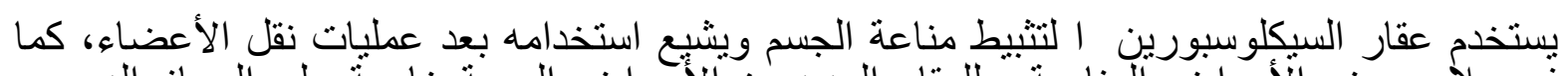

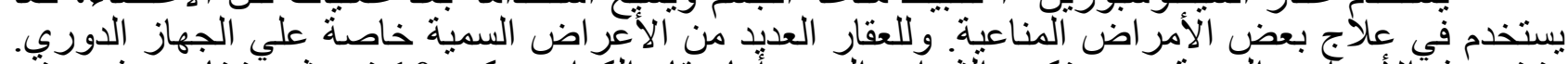

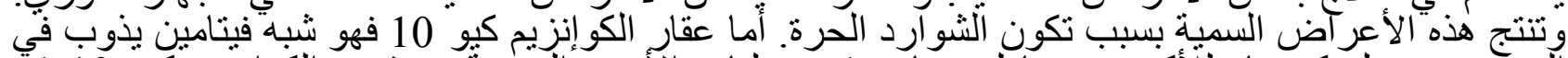

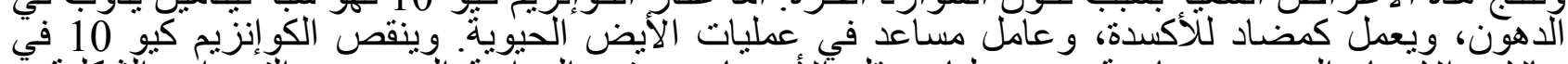

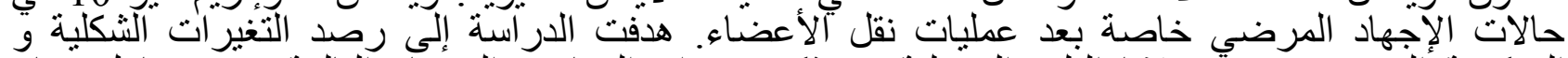

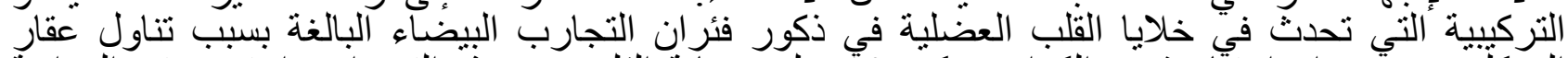

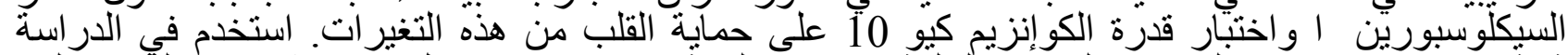

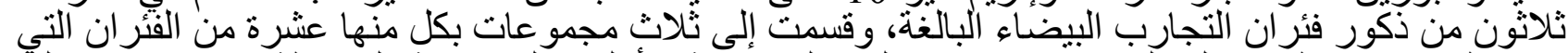

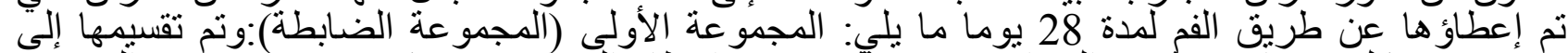

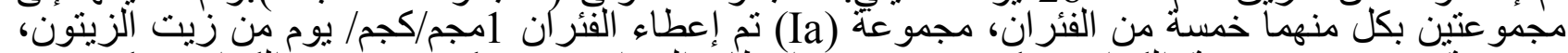

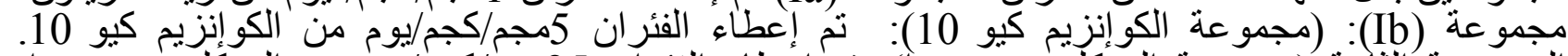

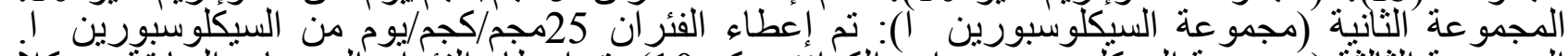

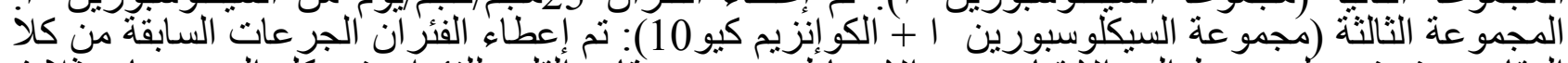

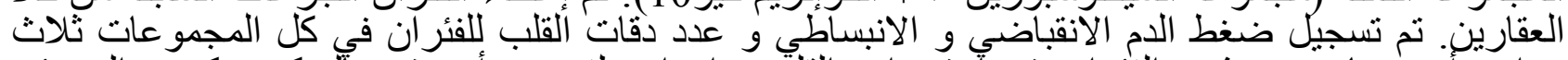

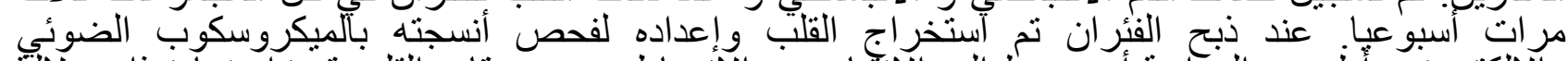

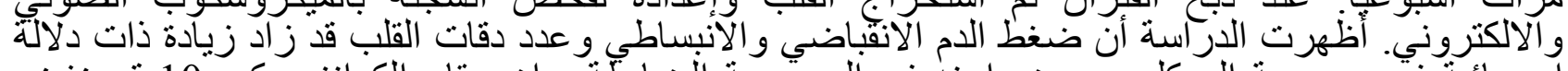

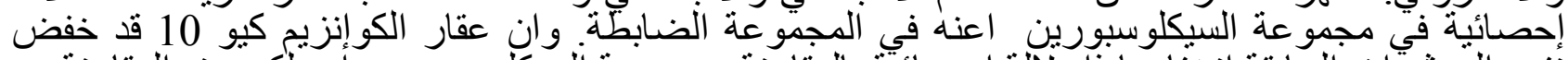

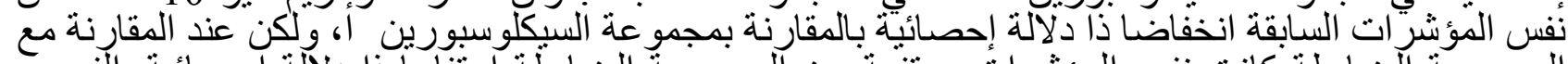

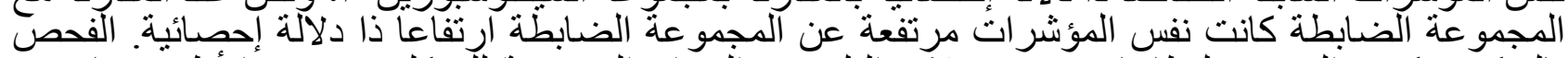

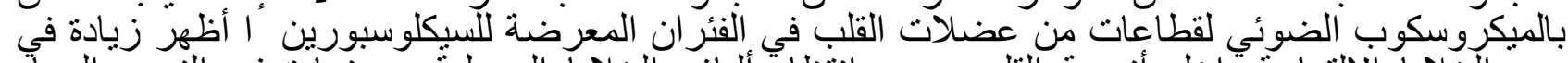

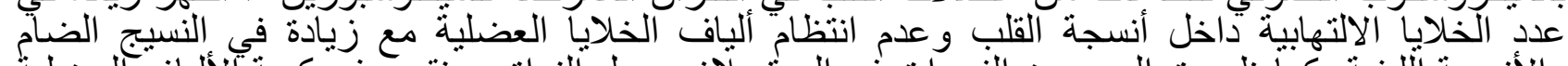

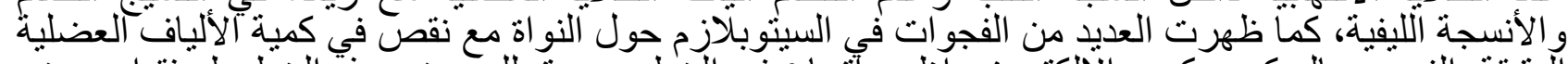

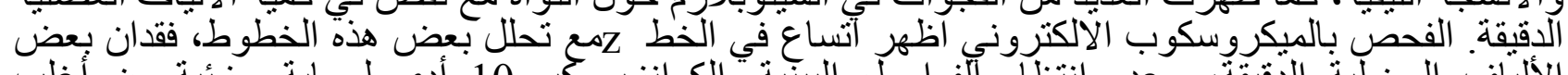

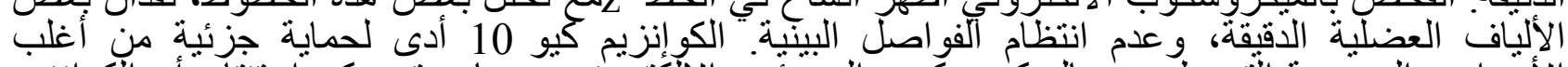

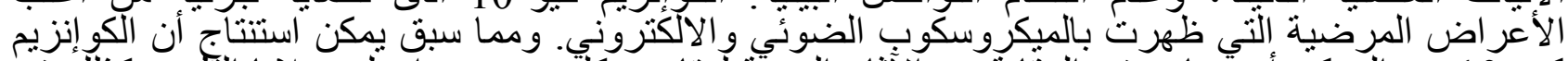

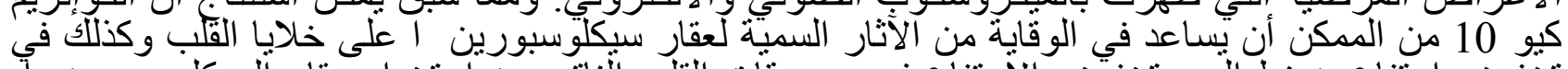

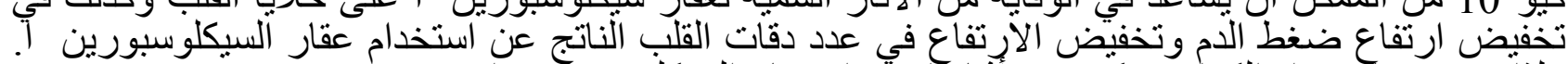

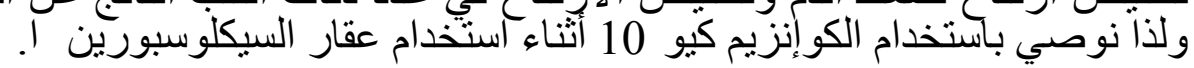

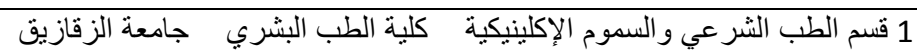

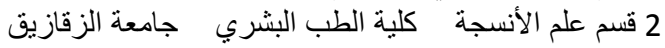

3 قسم علم الأدوية كلية الطبة الطبية البشري جامعة الزقازيق الزفازيق 